\title{
Factors affecting rectal temperature in infancy
}

\author{
C S Tuffnell, S A Petersen, M P Wailoo
}

\begin{abstract}
The recordings of 1197 overnight rectal temperatures from infants of up to 24 weeks of age have been analysed with respect to 12 variables, including a number of risk factors for sudden infant death syndrome. Multivariable regression was used to identify if parental smoking, bottle feeding, sleeping position, and birth weight affect the overnight rectal temperature of infants.

The rectal temperature, averaged over the period from three to five hours after the infants were put to bed, correlated well $(R=0.36)$ with the collected variables. An increase in the infant's age, birth weight, and the supine sleeping position all decreased the night time rectal temperatures. However, an increase in the night time room temperature, weight, and the combination of bottle feeding and parental smoking produced an increase in rectal temperature. The individual effects of bottle feeding and parental smoking were not significant. The results show that some of the major risk factors have the effect of raising the rectal temperature of sleeping infants.
\end{abstract}

(Arch Dis Child 1995; 73: 443-446)

Keywords: sudden infant death syndrome (SIDS), body temperature, passive smoking, sleeping position.

Epidemiological studies have identified numerous factors which increase the risk for sudden infant death syndrome (SIDS). ${ }^{12}$ Of these, some of the most important risk factors are the prone sleeping position, parental smoking, low birth weight, and possibly non-breast feeding. In addition, SIDS has been shown to somehow be related to temperature as SIDS occurs more often at higher latitudes in temperate areas $^{3}$ and in cooler months of the year. ${ }^{45}$ Overheating may also be a contributing factor for SIDS. ${ }^{67}$ However, the physiological link between temperature and SIDS has not been established.

When infants are put to bed their rectal temperature drops by up to $1^{\circ} \mathrm{C} .{ }^{8-10}$ For newborn infants this drop is very small, but increases until the rectal temperature falls to below approximately $36 \cdot 5^{\circ} \mathrm{C}$ each night. This is the mature or adult-like temperature pattern. ${ }^{10}$ However, many factors other than age contribute to the observed pattern of the rectal temperature. The aim of this research is to examine the effects of the main risk factors for SIDS on the night time rectal temperatures of infants.
Subjects and methods

From 1988 to 1994 , more than 500 infants in Leicestershire have been recruited for overnight studies of their body temperatures. Starting during the first few weeks of each infant's life, overnight recordings of body temperatures were typically made once a week for up to 15 weeks. At the time of recruitment, basic perinatal data were collected and for each overnight recording the age, weight, togs (thermal insulation of clothing and bedding), sleeping position, and state of health of the infant were recorded. For each recording, probes were attached to the infant to measure the skin and rectal temperatures. The temperatures of the room where the infant was sleeping were also recorded. The data were collected on a Grant Squirrel data logger, sampling at one minute intervals with a resolution of $0.05^{\circ} \mathrm{C}$.

From within this large database, all recordings where the infants were suspected of being ill or incubating an illness, or where the infants had recently had an immunisation, were removed. Also, only recordings where the probes remained attached for the entire night and the data were unblemished were considered for this analysis. Of the 159 recordings which were removed, there was no significant difference in distributions of the age, weight, togs, sleeping position, feeding regimen, and parental smoking when compared with the same variables in the original dataset. The remaining data were then prepared for subsequent analysis and included 1197 overnight recordings from 381 infants.

For comparison with the independent variables, the rectal temperature was averaged over the period from three to five hours after the infant was put to bed. This removed the large bias caused by the fall in rectal temperature which occurs when infants are put to bed. After five hours, a large percentage of the infants wake up and the rectal temperature becomes more variable.

The independent variables of age, gender, birth weight, weight at recording time, growth rate, feeding regimen, parental smoking (one or both parents); combined feeding and parental smoking, togs, average room temperature, and sleeping position were analysed with respect to the dependant variable rectal temperature. Multivariable regression and correlation was used to identify which variables significantly affect the infants' rectal temperature, ${ }^{11}$ while allowing for the possible confounding effects of all the variables. Dichotomous variables such as parental smoking were given the value of -1 (no or false) and +1 (yes or true). For the feeding regimen variable, the bottle feeding group included only those infants who were not breast fed at 
Results of multivariable regression with the rectal temperature averaged from three to five hours after bedtime. The mean (SD) of each variable is included; $\beta$ is the standard normal $z$ score regression coefficient, $b$ is the raw score regression coefficient, and $p$ is the two tail Student's t test

\begin{tabular}{lcccl}
\hline Variable & Mean (SD) & $\beta$ & $b$ & $p$ \\
\hline Age (weeks) & $12.1(4.58)$ & -0.432 & -0.0270 & $1.07 \mathrm{E}-16$ \\
Gender (female=-1, male=1) & $0.0159(1 \cdot 00)$ & -0.040 & -0.0115 & 0.163 \\
Birth weight (g) & $3212(696)$ & -0.194 & $-7.989 \mathrm{E}-5$ & $1.25 \mathrm{E}-5$ \\
Weight (g) & $5708(1233)$ & 0.231 & $5.374 \mathrm{E}-5$ & $1.45 \mathrm{E}-4$ \\
Growth rate (per day) & $0.00552(0.00432)$ & 0.018 & 1.1729 & 0.617 \\
Feeding (breast=-1, bottle=1) & $0.402(0.916)$ & -0.036 & -0.0112 & 0.328 \\
Parental smoking (no=-1, yes=1) & $0.021(1.00)$ & -0.077 & -0.0223 & 0.193 \\
Bottle feeding and parental & & & & \\
$\quad$ smoking (no=-1, yes=1) & $-0.114(0.994)$ & 0.160 & 0.0463 & 0.019 \\
Togs & $10.5(4.01)$ & 0.001 & $8.8598 \mathrm{E}-5$ & 0.967 \\
Room temperature $\left({ }^{\circ} \mathrm{C}\right)$ & $20.5(2 \cdot 46)$ & 0.061 & 0.0071 & 0.046 \\
Supine (no=-1, yes=1) & $-0.123(0.993)$ & -0.101 & -0.0290 & 0.017 \\
Prone (no=-1, yes=1) & $-0.529(0.849)$ & -0.024 & -0.0080 & 0.522 \\
\hline
\end{tabular}

all. Thus, breast feeding included combined breast and bottle feeding. Growth rate was calculated on a weekly basis then divided by 7 and also divided by the weight to give the daily growth rate per unit mass. Two tail Student's $t$ tests were used to indicate the significance of the partial regression coefficients such that the number of samples was taken to be the number of infants, not the number of recordings.

\section{Results}

Multivariable regression and correlation of the 12 independent variables is presented in the table. The correlation coefficient of the multivariable regression was $R=0.36$ and the raw score regression constant was $\mathrm{k}_{0}=36 \cdot 777^{\circ} \mathrm{C}$. The values of $\beta$ are the standard normal z score regression coefficients which indicate the relative effect of each of the independent variables on the rectal temperature. The raw score regression coefficients are represented by the $b$ values. The values of $p$ give the significance of the regression coefficients with two tail Student's $t$ tests. The mean (SD) of the rectal temperature was $36 \cdot 65(0 \cdot 286)$.

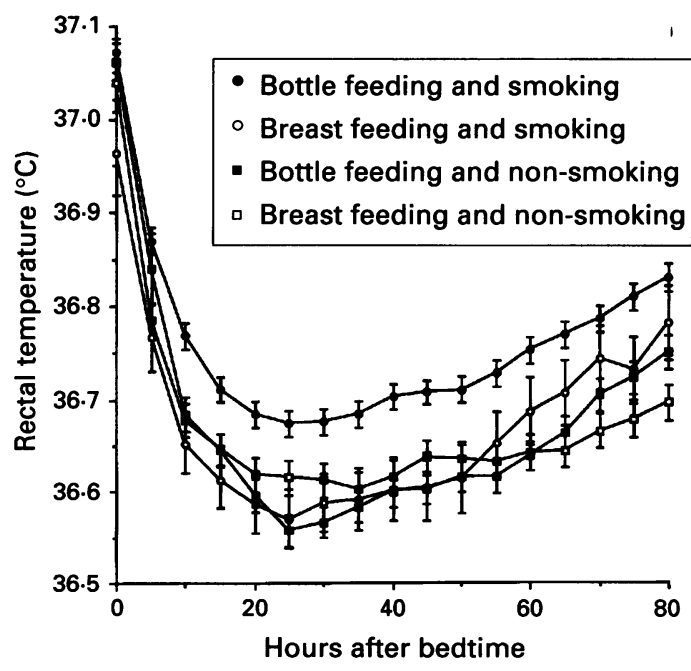

Figure 1 Effect of the four combinations of feeding regimen and parental smoking on the night time rectal temperatures of infants. Data have been averaged across all infants in each of the four groups and the error bars indicate the standard error. The first eight hours of rectal temperatures after the infants have been put to bed is shown. All variables, excluding parental smoking and feeding regimen, were sufficiently similar to allow presentation of these data on a univariate graph.

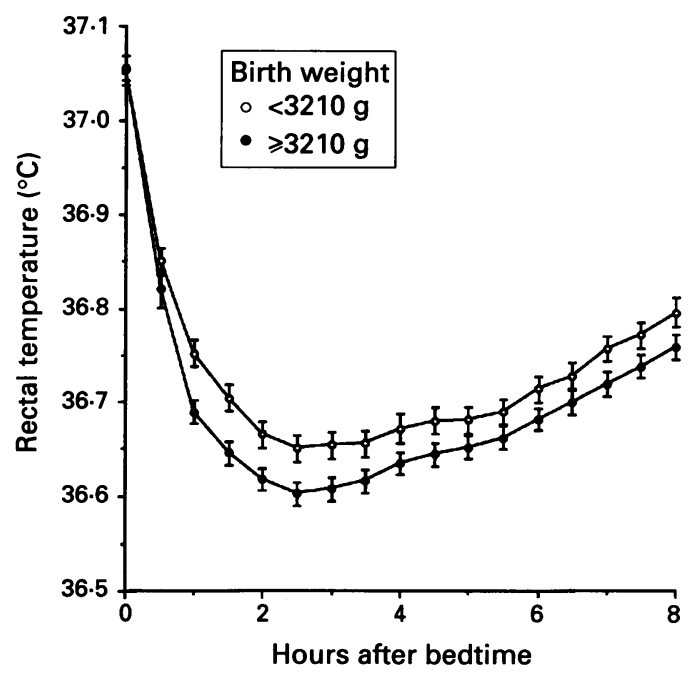

Figure 2 Effect of birth weight on the night time rectal temperatures of infants. Data have been averaged across all infants in the low birthweight and high birthweight groups and the error bars indicate the standard error. The first eight hours of rectal temperatures after the infants have been put to bed is shown. All variables, excluding birth weight, were sufficiently similar to allow presentation of these data on a univariate graph.

From the values of $\beta$ and $p$ presented in the table, the infant's age has by far the largest and most significant effect on the rectal temperature. As the infants mature, the average rectal temperature falls, matching previous results reported in the literature. ${ }^{10}$ The supine sleeping position also significantly lowers the rectal temperature, agreeing with previous research. ${ }^{12}$

The individual effects of either bottle feeding or parental smoking are not significant. However, the combined effect of bottle feeding and parental smoking is significant, the effect of which is approaching half the effect of age. Figure 1 shows the effects of smoking and bottle feeding on the rectal temperature, averaged over the groups within the entire dataset. The three rectal temperature curves of breast feeding plus parental smoking, breast feeding without parental smoking, and bottle feeding without parental smoking are statistically indistinguishable. However, the curve for bottle feeding plus smoking is approximately $0.1^{\circ} \mathrm{C}$ higher than the other three curves. This univariate result agrees with that presented in the table. In addition to the results presented in the table, the correlation between the bottle feeding and parental smoking variables was $r=0.37(\mathrm{p}<0.0001)$.

Body weights also have a significant effect on the average rectal temperature. A low birth weight raises the rectal temperature, contrasting with a low body weight, measured at the time of the recording, which lowers the rectal temperature. The effect of birth weight on rectal temperature is presented in fig 2 . The data have been split into two groups, which are birth weight less than and greater than or equal to the mean birth weight of $3210 \mathrm{~g}$. In agreement with the table, this graph shows that the lower birthweight group has a rectal temperature which is approximately $0.05^{\circ} \mathrm{C}$ higher than the high birthweight group.

The only other significant variable is the average room temperature. An increase in the 
room temperature of $10^{\circ} \mathrm{C}$ produces an increase in the rectal temperature of $0.07^{\circ} \mathrm{C}$. Although this effect is significant, it is relatively small.

\section{Discussion}

Because of the large number of infants studied, it is not likely that the significance of the variables listed in the table will change with the addition of data from more infants. However, these variables by no means account for the entire variability of the rectal temperature. Considering the feeding and smoking variables in this study are dichotomous, when their effect is likely to be dose related, and the limited accuracy of response to questionnaires required to gather the data, the multivariable correlation coefficient of 0.36 is more than satisfactory. Also, the analysis presented in the table assumes linear relationships, but for all except true dichotomous variables such as gender, this is not likely to be the case.

The results suggest that low birthweight infants have a raised sleeping rectal temperature throughout early life. As growth rate had no effect on rectal temperature, it would seem that a higher metabolism in the lower birthweight infants during the period when their weight is 'catching up' with other infants, is not the cause of the increased rectal temperature. The effect of body weight (measured at recording time) on rectal temperature has the opposite effect of birth weight. In this result, a higher body weight infant has a higher rectal temperature. Typically, an infant's weight increases with age and these results show that older infants have a lower rectal temperature, which highlights the result that the effect of body weight is independent of age. Therefore, the elevating effect of increased body weight on rectal temperature probably reflects the increased insulation or surface area to volume ratio of infants with a greater weight.

The results also indicate that parental smoking and bottle feeding combine to raise the infant's rectal temperature. One possible explanation for this peculiar behaviour is that infants who were bottle fed and whose parents were smokers were more likely to be incubating a mild illness which was not detected by the parents. However, as all data from infants who were known to be ill were removed from the analysis, there is no direct evidence of illness affecting the rectal temperatures. The results also indicate that, as parental smoking only affects an infant's rectal temperature when the infant is not breast feeding, the effect of parental smoking is not through breast milk but is likely to occur either in utero or through passive smoking. From the data collected on maternal smoking habits, it was impossible to establish if the effect of parental smoking occurred in utero or through passive smoking. The relatively strong correlation between bottle feeding and parental smoking $(r=0.37)$ highlights the difficulty of isolating the effects of parental smoking habits and feeding regimen for studies with low numbers of samples, thus contributing to the uncertainty of the importance of bottle feeding as a possible risk factor for SIDS. However, as a synergetic relationship was also identified for bottle feeding and parental smoking in this study, it may prove interesting to examine this relationship in other areas of research.

A lowering of the rectal temperature of supine sleeping infants may be a direct result of the infant's ability to lose heat from the skin. An infant lying prone or laterally is likely to have the highly radiative region of the face partially insulated against the mattress. In contrast, supine sleeping infants may be able to move more freely from under the bed clothes and use hands for cooling when they feel too hot. However, the validity of these hypotheses needs to be tested.

Although the average room temperature has only a small effect on the rectal temperature, it remains significant such that a hot room increases the rectal temperature. However, the data were collected for normal changes in room temperature (mean 20.5, SD 2.46) and therefore cannot be used to predict the effects of large temperature changes. Another environmental influence is the tog level which is not significant. The lack of significance of tog level is probably a result of two factors. Firstly, the measurement of tog level is not very accurate as the tog values can only be estimated, not measured, for each item of bedding and clothing. Secondly, the tog levels do not in any way account for heat loss through the uninsulated skin of the face and in some cases the hands. The effect of small changes in insulation on heat loss is likely to be overshadowed by the highly variable fraction of uninsulated skin that loses heat considerably more rapidly than clothed skin. As a supine sleeping infant can easily move the bedding, the true level of insulation cannot be accurately assessed over an entire night.

Of the variables listed in the table which affect the rectal temperature two are direct and major risk factors for SIDS. Both low birth weight and non-supine sleeping raise the rectal temperature of sleeping infants. Although parental smoking, which is another major risk factor for SIDS, did not directly affect the rectal temperature, the combination of parental smoking and bottle feeding significantly raised the rectal temperature. Bottle feeding has not consistently been recognised as a risk factor for SIDS in the UK. However, in New Zealand bottle feeding proved to be an important risk factor. ${ }^{2}$ Gender is also an important risk factor in that male infants have an increased risk for SIDS. ${ }^{1}$ However, these results found no link between gender and the average night time rectal temperatures of infants.

The predicted change in rectal temperature that occurs, when the variables in the table which significantly affect the rectal temperature come together, is calculated by summing the products of the $b$ values of each variable with the change in the associated variable from low to high risk for SIDS. For the birthweight variable, the low to high risk change has been chosen as $2 \mathrm{SD}$ and for dichotomous variables 
the change value is two (the difference between the input values of -1 and 1 ). Although the combined parental smoking and bottle feeding variable is not directly related to SIDS, it is included here because independently, smoking and possibly breast feeding are important. The resulting increase in the night time rectal temperature, which occurs when these factors come together, is $0.26^{\circ} \mathrm{C}$.

The results suggest that some of the risk factors for SIDS have an effect that raises the rectal temperature of sleeping infants. None of the SIDS risk factors lowered the rectal temperature. None of the infants studied subsequently died from SIDS, therefore no direct relationship between SIDS and increased rectal temperature could be established. In addition, the effect of the variables studies on the rectal temperature appears to be small yet it accounts for 0.93 of $1 \mathrm{SD}$ of the rectal temperature. Moreover, an infant who succumbs to SIDS may have an increased sensitivity or may have developed under extreme conditions of the variables which affect the core body temperature, thus the factors which affect the infant's rectal temperature may remain important. Further research is required to investigate these speculative hypotheses.

Dr Tuffnell was supported by the Foundation for the Study of Infant Deaths, Leicester Medisearch, and the Cot Death
Division of the National Children's Health Research Foundation of New Zealand. The original data were collected by a number of research health visitors associated with the Leicester team, all of them are thanked for their cooperation with the analysis.

1 Engelberts A. Cot death in the Netherlands: an epidemiological study. Amsterdam: VU University Press, 1991. (MD thesis.)

2 Mitchell EA, Taylor BJ, Ford RPK, et al. Four modifiable and other major risk factors for cot death: the New Zealand study. $¥$ Paediatr Child Health 1992; 28 (suppl 1): Zealand

3 Nelson EAS, Taylor BJ. Climatic and social associations with postneonatal mortality rates within New Zealand. NZ Med f 1988; 101: 443-6.

4 Murphy MFG, Campbell MJ. Sudden infant death syndrome and environmental temperature: an analysis using vital statistics. I Epidemiol Community Health 1987; 41: 61-71.

5 Campbell MJ. Sudden infant death syndrome and environmental temperature: further evidence of a time-lagged relationship. Med f A A ust 1989; 151: 365-7.

6 Ponsonby AL, Dwyer T, Gibbons LE, Cochrane JA, James ME, McCall MJ. Thermal environment and sudden infant death syndrome: case control study. $B M \mathcal{F} 1992$; 304: 277-82.

7 Nelson EAS, Taylor BJ, Weatherall II. Sleeping position and infant bedding may predispose to hyperthermia and the sudden infant death syndrome. Lancet 1989; i: the sudden

8 Wailoo MP, Petersen SA, Whitaker H, Goodenough P. Sleeping body temperatures in 3-4 month old infants. Sleeping body temperatures in

9 Brown PJ, Dove RA, Tuffnell CS, Ford RPK, Oscillations of body temperature at night . Arch Dis Child 1992; 67: 1255-8.

10 Lodemore MR, Petersen SA, Wailoo MP. Factors affecting the development of night time temperature rhythms. Arch Dis Child 1992; 67: 1259-61.

11 Overall JE, Klett CJ. Applied multivariate analysis. New York: McGraw-Hill, 1972: 415-40.

12 North RG, Petersen SA, Wailoo MP. Lower body temperature in sleeping supine infants. Arch Dis Child 1995; 72 340-2. 